\title{
Puncture of the Maxillary Sinus: When, How and Why?
}

\author{
Novak Vukoje* and Dr Jon Garito \\ Department of ENT Surgery, Novi Sad, Serbia
}

Submission: July 30, 2018; Published: August 03, 2018

*Corresponding author: Novak Vukoje, Department of ENT Surgery, Novi Sad, Serbia, Email: drvukoje@hotmail.com

\begin{abstract}
Background: The author has analyzed the success of the puncture in the treatment of the chronic purulent maxillary sinusitis in 43 patients, aged from 19 to 51, previously diagnosed (RTG PNS, CT, NMR) and treated longer than 3 months without a significant improvement. Sense of pressure on the side of the affected sinus (76\%), post nasal drip (71.8\%), halitosis (47.9\%), nasal obstruction (46.2\%), impaired smell, sub febrile temperature, fatigue etc., dominated the symptomatology. The duration of symptoms varied from 6 months to two years. The intervention of sinus puncture was carried out through the lower nasal passage, during one or more sessions of secretion aspiration, sinus lavage and drug administration in the above-mentioned passage. Puncture specimens of all the patients were sent for bio gram and antibiogram analysis. No complications were noticed. The goal of this work is to evaluate the adequacy of applying the puncture as a medical procedure in the treatment of the chronic purulent inflamed maxillary sinus.

Results: healing or substantial improvement rate of the process on the maxillary sinuses after merely one puncture was $60.7 \%$ and $80 \%$ after two or over three procedures.

Conclusion: The author emphasizes the necessity of applying the puncture in certain diseases of the maxillary sinus, especially in sinus empyema when an immediate secretion removal is needed, and when the puncture can prevent the development of endo- or extracranial complications. The puncture can come to great use in all conditions where there is simultaneous presence of the sinus disease with secretion and other chronic diseases of the lower respiratory tract (asthma, chronic bronchitis).
\end{abstract}

\section{Introduction}

Chronic sinusitis is the most common disease in the USA with about 37 million people affected which comprises 14.13 $\%$ of the population, meaning that every seventh person suffers from the disease. This impairment occurs more frequent than arthritis (12.4\%) and hypertension (11.44\%) and is defined as an inflammatory disease of the nasal mucosa with symptoms that last longer than three months. Those dominant symptoms are: sense of pressure on the side of the affected sinus, post nasal drip, bad breath, nasal obstruction, impaired smell, fatigue and sub febrile temperature. The maxillary and ethmoid sinus are most frequently affected. In over $40 \%$ of the maxillary sinus inflammations, the process also involves the front ethmoid cells. Sinusitis can present itself on one or more sinuses (polysinusitis, pansinusitis) and is classified by the course of the disease into acute, subacute and chronic sinusitis.

The seasonal character of the sinusitis in not seldom associated with an outbreak of allergens who are more present in springtime and in the fall and with a viral infection in winter period. Chronic maxillary rhinosinusitis, particularly in the state of exacerbation, could present a potential threat for the development of the endocranial and extracranial complications. A late diagnosis and an inadequate treatment are most frequently stated as the reasons for these complications. The normal drainage of the maxillary sinus occurs through hiatus semilunaris into the middle nasal meatus. The mucosal drainage canal is 6-8 mm long, 3-5 mm wide and is located on the roof of the sinus which impedes the drainage. The capacity of the maxillary sinus and its excretory duct is often disproportionate, especially in the state of an infection. That is when a build-up of a pathological content occurs which cannot be spontaneously eliminated for two reasons: a blocked excretory canal or a dyskinesia of the epithelium. As a means of treating and faster secretion evacuation, the puncture of the sinus as a procedure was introduced in 1886. by dr. Mikulicza.

Depending of the purpose, the puncture can be classified into:
a. Diagnostic puncture
b. Prophylactic puncture
c. Therapeutic puncture 


\section{Global Journal of Otolaryngology}

Regarding the site of performing the procedure, the puncture is divided into:

a. Frontal (through the fossa canina)

b. Lower (under the inferior nasal concha)

c. Middle (through the natural drainage ostium)

In the case where a perforation of the lateral nasal wall is not possible for any reason (thickness of the medial wall of the sinus, the size of the sinus- shallow, undeveloped, septate etc., nasal pathologies (septum deviation, polyps, synechia, enlarged nasal concha etc.) etc. trepanation can be carried out through the frontal wall of the maxillary sinus, through fossa canina. By puncturing the sinus, because of the fast elimination of the pathological content, we can prevent the spreading of the process into the adjacent regions.

This does not require any special patient preparation and it is performed by ENT specialists on their surgical practice premises. After administrating an epimucose anesthesia to the nasal mucosa, a needle is placed on the border of two front thirds and back third of the nasal concha, around $1 \mathrm{~cm}$ above the nasal floor in the direction of the lateral eye angle on the same side. The thin bone lamella of the medial wall of the sinus is perforated with a needle. As a next step, aspiration is performed and sinus lavage and, if needed, placing a medicine in the sinus. When the condition requires a continuous drainage and sinus ventilation, other surgical procedures are recommended.

Today's trends suggest FESS (Functional Endoscopic Sinus Surgery) which requires hospital treatment and demands special and costly equipment, strictly defined indications and the adequate training of the physicians for its performing. Nevertheless, the rating of other surgical procedures, such as the "antral window" procedure, is in downfall because of the FESS. Prior to any intervention, one must determine the size and the content of the sinus. For that purpose, we can use methods such as CT, NMR or even a regular RTG of the paranasal cavities can come to use. Occasionally, a clinical check up with the anamnestic data and an RTG of the paranasal cavities is enough to make a diagnosis.

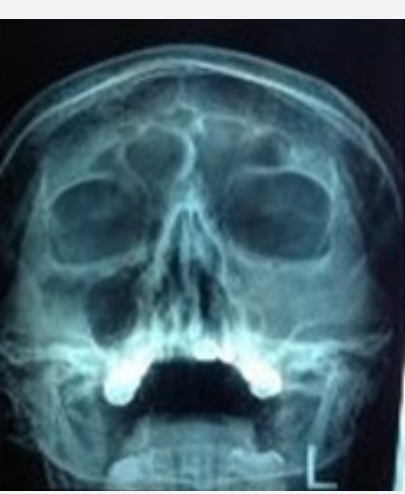

Figure 1: Diffusely shadowed left maxillary sinus.
The maxillary sinus is piramide-shaped

a. Width $23-25 \mathrm{~mm}$

b. Height $30-33 \mathrm{~mm}$

c. Anteroposterior dimension $32-34 \mathrm{~mm}$

d. Volume 16-18ml (Figures 1-3)

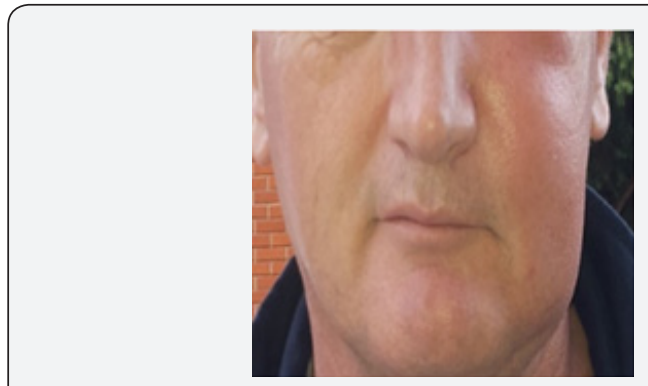

Figure 2: Local medical finding of the same patient with an exacerbation of the chronic purulent sinusitis.

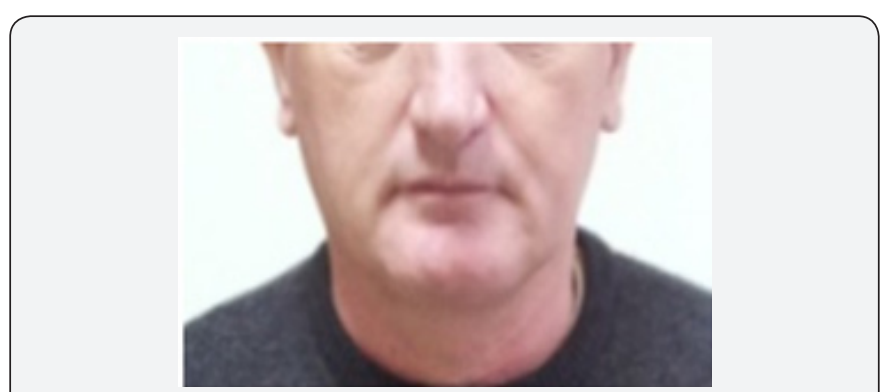

Figure 3: Local finding second day after the puncture, lavage and insertion of antibiotics into the sinus.

Since the two-week antibiotics treatment (longaceph) did not lead to healing of the process, the sinus has been punctured (Figure 4).

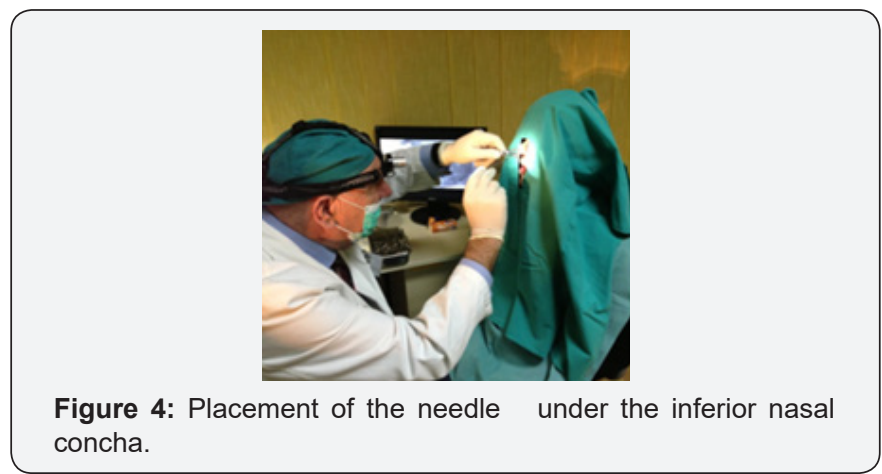

The sinus puncture is considered when:

1. When the medication treatment of acute, subacute or chronic suppurative inflammation of the maxillary sinus didn't produce a favorable outcome

2. With patients who have a sinus pathology and a simultaneous pathology of the lower respiratory tract (secretion immobility) 
3. When a patient is at risk of complications due to his/ her sinus disease

4. For the collection of specimens for microbiological and pathohistological analyses

5. In cases when a condition requires a direct insertion of a drug into the sinus

6. When a surgery is contraindicated

7. Patient does not wish it (Figures 4-7)

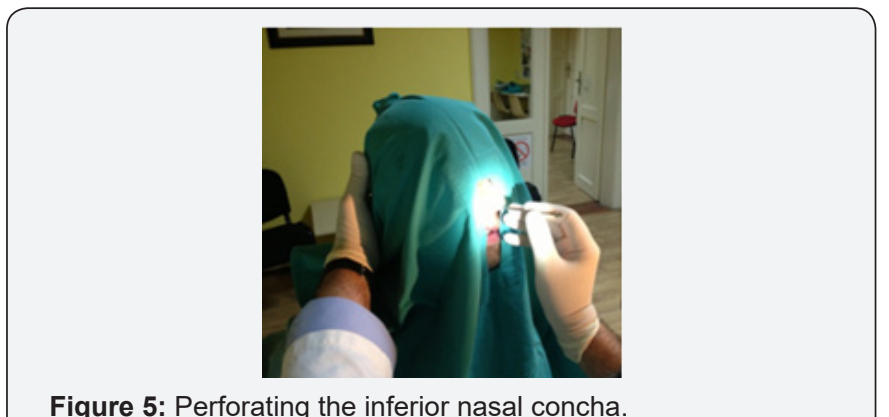

Figure 5: Perforating the inferior nasal concha.

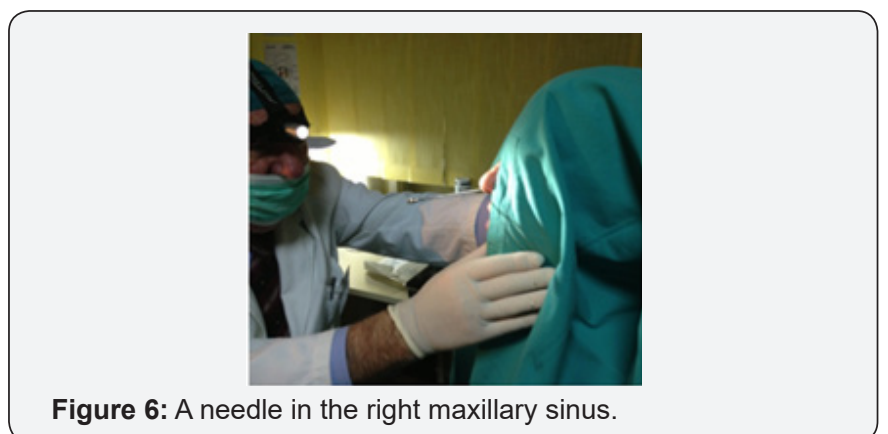

Figure 6: A needle in the right maxillary sinus.

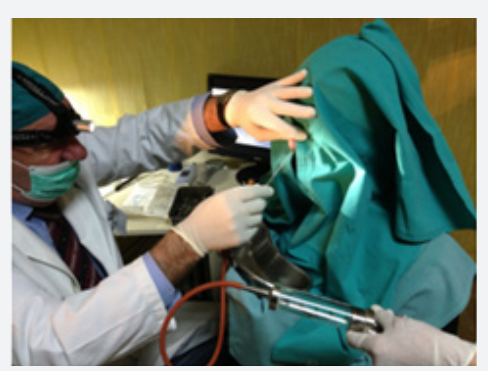

Figure 7: Maxillary sinus lavage.

In carefully chosen cases the procedure will prove itself to being favorable in the treatment of mucopurulent maxillary rhinosinusitis and should not be avoided. The evacuation of the pathological content of the sinus will contribute greatly to the healing and reactivating natural sinus drainage. Puncture can be performed at the office and doesn't require hospitalization. If the natural opening of the maxillary sinus is shut or blocked, a pain should occur with the injecting of a liquid into the sinus. In that case, we pause the intervention, to insert another needle, next to the existing one, through which the sinus content is to be evacuated. A cause for a negative puncture can be, stuffed needle, a needle in contact with the back wall of the sinus, needle in a sinus polyp or tumor mass, thick, caseous content, etc.

\section{Puncture complications:}
a. Eye injury
b. Soft tissue of the cheek injury
c. Infection entry point
d. Hemorrhage
e. Emphysema of the cheek and lower eyelid
f. Damage to the nasolacrimal duct

The rate of occurrence of these complications is $4 \%$, and they are often a result of a lack of skill of the doctor, altered anatomy, inadequacy of the technique involved, etc.

\section{Disadvantage of the Puncture}
a. The sinus must be punctured several times
b. Oftentimes the procedure gives temporary relief
c. Doesn't prevent a recurrence of the disease or new infections

\section{d. Must be combined with the antibiotic treatment}

In the case when the puncture doesn't prove itself successful, other procedures should be performed. One of the most frequently performed procedures from the group "antral window" procedures is nasoantrostomy and meatotomy. They imply creating an opening on the lateral wall of the nose below the nasal concha and establishing a wider communication between maxillary sinus and inferior nasal passage.

However, indications for this intervention as well as curettage, that is the removal of the affected mucosa, remains questionable. Numerous authors give priority to drainage rather than curettage, "drainage prime le curettage". It needs to be mentioned, that the fenestration represents unnatural way of drainage and sinus ventilation and facilitates the spreading of infection from the nose to the sinus, which is attributed as a disadvantage of the procedure. Moreover, spontaneous closure of the opening, before the healing process has terminated, can compromise the success and credibility of these procedures. Postoperative course could require nasal douche and sinus rinsing with a prescribed antibiotic therapy. These interventions are practically without complications and are indicated in patients who suffer from chronic maxillary rhinosinusitis with an immobile secretion as well as in recurring and frequent inflammations. It can also serve as a first step for the endoscopic evaluation of the sinus cavity, doing biopsies, continuous lavage of the sinus, dealing with headaches due to sinus disease, etc. Finally, these procedures are legitimate in cases where we lack results from the medial meatotomy (FESS). 


\section{Conclusion}

The author concludes that, the puncture of the sinus is adequate and justified in the treatment of chronic maxillary sinusitis with the presence of suppurative secretion where the use of medications has not solved the issue. The sinus empyema is an absolute indication for this procedure. When the recurring cases of chronic sinusitis are at issue, recurring sinusitis with secretion immobility, puncture could be a temporary solution. If the impaired sinus requires continuous drainage, advantage has to be given to other functional operations. Furthermore, puncture of the sinus is a method that must not be left out in patients who have a blockage of the osteomeateal complex which has not been resolved by FESS operation. By using the puncture, we remove the pathological content from the sinus, perform sinus lavage, drug insertion, and collection of the material for analyses, etc. The evacuation of the pathological content of the sinus will contribute greatly to the healing and reactivating natural sinus drainage. Puncture can be performed at the office and doesn't require hospitalization. Sinus puncture has the advantage as an urgent intervention in patients with endo- and extracranial complications. Minimum trauma, absence of serious complications, preservation of the integrity of the adjacent regions, the simplicity of the procedure makes this intervention unique, and in case of failure, there is always a possibility of another surgical procedure. In correctly chosen cases there is full medical justification for this form of treatment where the patients, even after a short period of time after the procedure, regained the capability in performing normal activities and fully recovered.

\section{Your next submission with Juniper Publishers will reach you the below assets}

- Quality Editorial service

- Swift Peer Review

- Reprints availability

- E-prints Service

- Manuscript Podcast for convenient understanding

- Global attainment for your research

- Manuscript accessibility in different formats

( Pdf, E-pub, Full Text, Audio)

- Unceasing customer service

Track the below URL for one-step submission https://juniperpublishers.com/online-submission.php 